\title{
Vaskuläre Effekte der alveolären Hypoxie - Sensor- und Signaltransduktionsmechanismen
}

\author{
Vascular Effects of Alveolar Hypoxy - Sensing and Signal Transduction \\ Mechanisms
}

\section{Förderpreis der}

\section{Deutschen Gesellschaft für Pneumologie 2002}

„Der Schwerpunkt der Preisarbeit von Herrn Dr. Norbert Weißmann liegt auf Untersuchungen zur hypoxischen pulmonalen Vasokonstriktion (HPV, von Euler-Liljestrand-Mechanismus). Mit der Arbeit konnte der Preisträger ein neues Konzept von der Regulation dieses Mechanismus entwickeln, das reaktive Sauerstoffspezies als Vermittler der HPV in das Zentrum stellt. Die Arbeit leistet somit einen wesentlichen Beitrag zum Verständnis der Sensor- und Signaltransduktionsprozesse der hypoxischen pulmonalen Vasokonstriktion und des vaskulären Sauerstoffsensings. Ohne Zweifel gehört der Preisträger mit seinen Arbeiten der vordersten Reihe der Wissenschaftler an, die das Thema der pulmonalen Vasoregulation bearbeiten.“

Prof. Dr. Werner Seeger, Gießen
Die Struktur der menschlichen bzw. der Säugetierlunge ermöglicht einen äußerst effizienten Gasaustausch. Dieser wird durch eine große Oberfläche und eine dünne Diffusionsbarriere zwischen Alveolar- und Kapillarraum der Lunge ermöglicht. Neben den anatomischen Voraussetzungen bedarf es jedoch auch einer physiologischen Regulation, um einen optimalen Gasaustausch sicherzustellen. Notwendig ist eine solche Regulation aufgrund der Tatsache, dass auch in der gesunden Lunge unzureichend oder nicht ventilierte Lungenareale auftreten. Der Blutfluss zu solchen minderbelüfteten (hypoxischen) Lungenarealen wird gedrosselt, um eine Anpassung der Perfusion an die Ventilationsbedingungen vorzunehmen. Die Drosselung geschieht durch im Wesentlichen präkapilläre Vasokonstriktionen. Der Blutfluss wird durch diesen Regulationsvorgang aus unzureichend ventilierten (hypoxischen) Lungenbezirken ferngehalten und in ausreichend ventilierte Lungenareale umgeleitet. Dieses Prinzip der Ventilations-Perfusions-Anpassung wird als „hypoxische pulmonale Vasokonstriktion (HPV)“ bezeichnet. Die moderne Ära der Untersuchung dieses Regulationssystems geht auf Experimente durch U. S. von Euler und G. Liljestrand aus dem Jahre 1946 zurück. Der Mechanismus der „hypoxischen pulmonalen Vasokonstriktion“ ist aus diesem Grund auch unter dem Begriff „Von Euler-Liljestrand-Mechanismus“ in die Literatur eingegangen.

Pathophysiologisch von Bedeutung ist

1. eine Störung dieses Mechanismus und

2. die Auswirkung einer generalisierten alveolären Hypoxie auf die Lunge.

Störungen des akuten Regulationsmechanismus können eine arterielle Hypoxämie zur Folge haben, wie sie im Zuge von Pneumonien, im Rahmen eines akuten Atemnotsyndroms des Er- 
wachsenen (ARDS) oder auch im Zusammenhang mit Leberzirrhosen beobachtet werden. Die generalisierte alveoläre Hypoxie führt den Mechanismus der hypoxischen Vasokonstriktion hingegen ad absurdum, indem unter diesen Bedingungen in nahezu allen Lungenarealen Vasokonstriktionen auftreten, die zu einer Verringerung des Gesamtgefäßquerschnitts der pulmonalen Strombahn führen und über den so erhöhten pulmonalvaskulären Widerstand einen Anstieg des pulmonalarteriellen Druckes mit Rechtsherzbelastung zur Folge haben.

Hält die generalisierte Hypoxie über Wochen bis Monate an, wird die pulmonale Hypertonie durch einen Gefäßumbauprozess, der im Wesentlichen durch eine Mediahypertrophie und de novo Muskularisation der pulmonalen Blutgefäße gekennzeichnet ist, fixiert. Als Konsequenz der hohen pulmonalvaskulären Widerstände kann dieses pathophysiologische Geschehen in einem Cor pulmonale gipfeln.

Obwohl die Untersuchungen durch von Euler und Liljestrand bereits auf das Jahr 1946 zurückgehen, sind bis heute die Natur und Lage der Sauerstoffsensoren in der Lunge und die Signaltransduktion, die zum einen zur Vasokonstriktion unter alveolärer Hypoxie führen und zum anderen den Gefäßumbauprozess unter chronischer Hypoxie bedingen, nur lückenhaft geklärt. Ziel der Arbeit war es, diese Regulationsprinzipien zu untersuchen.

In den hierzu durchgeführten Untersuchungen wurde die HPV zunächst in dem Modell der isoliert ventilierten und perfundierten Kaninchenlunge charakterisiert. In diesen Experimenten gelang es, die Lokalisation des $\mathrm{O}_{2}$-Sensors auf Bezirke der Lunge einzugrenzen, die in ihrem $\mathrm{pO}_{2}$ nur durch den Sauerstoffpartialdruck des alveolären Gases bestimmt sind. Die Charakterisierung ergab weiterhin, dass die Vasokonstriktionen im Wesentlichen präkapillär lokalisiert sind. Der Verlauf der Gefäßkontraktion unter alveolärer Hypoxie zeigte eine biphasische Kinetik, mit einer sehr raschen initialen Reaktion innerhalb von wenigen Sekunden, die nach Erreichen eines Maximums nach ca. 5-6 Minuten und einer nachfolgenden Abschwächung durch einen protrahierten Anstieg des pulmonalvaskulären Widerstandes über die Untersuchungsperiode von 3 Stunden gekennzeichnet war. Interessanterweise war diese Widerstandserhöhung nach Beendigung einer 2-3stündigen hypoxischen Beatmungsphase, im Gegensatz zu einer nur 10-minütigen hypoxischen Ventilationsphase, nicht mehr vollständig reversibel. Aufgrund der Untersuchungen konnte postuliert werden, dass bereits innerhalb weniger Stunden die Regulationsprozesse aktiviert werden, die zur Fixierung des pulmonalen Hypertonus unter chronischer Hypoxie führen. Darüber hinaus konnte gezeigt werden, dass die Regulationsprinzipien der frühen (akuten) Phase der hypoxischen Vasokonstriktion (Hypoxiedauer: $10 \mathrm{~min}$ ) und der protrahierten Vasokonstriktion (Hypoxiedauer: 2-3 Stunden) sowie der Erhöhung des posthypoxischen Vasotonus nach mehrstündiger alveolärer Hypoxie bezüglich der Beteiligung reaktiver Sauerstoffspezies und NO identisch zu sein scheinen. Eine Frage, die bisher in der Literatur für Untersuchungen intakter Lungen nicht bearbeitet war.

Um die der akuten Phase der hypoxischen Vasokonstriktion zugrunde liegenden Mechanismen zu charakterisieren, wurden Experimente mit pharmakologischer Beeinflussung potenzieller Sensor- und Mediatorsysteme sowie mit Bestimmung der Frei- setzung potenziell hypoxieregulierter Mediatoren vorgenommen. Die Daten weisen darauf hin, dass bezüglich des Arachidonsäuremetabolismus Cyclo- und Lipoxygenase-Produkte nicht spezifisch in die Regulation der akuten HPV involviert sind. Für Cytochrom $\mathrm{P}_{450}$-abgeleitete Arachidonsäuremetaboliten erscheint eine Beteiligung unwahrscheinlich, konnte jedoch nicht ausgeschlossen werden.

Untersuchungen zur Rolle von Stickstoffmonoxid ergaben, dass NO spezifisch in die Regulation der HPV involviert ist:

1. Unter alveolärer Hypoxie kam es zu einer selektiven Verringerung der exhalativen NO-Liberierung der Lunge, die zeitlich der pulmonalarteriellen Druckantwort vorausging, während die nach intravasal gerichtete NO-Freisetzung von einer alveolären Hypoxie nicht beeinflusst wurde.

2. Die pharmakologische Blockade der endogenen NO-Synthese führte zu einer Potenzierung der Stärke der HPV, die spezifisch für die hypoxieinduzierte Vasokonstriktion war, wie der Vergleich mit nicht-hypoxieinduzierten Vasokonstriktionen zeigte.

3. Die Anreicherung des Perfusionsmediums der isolierten Lungen mit NO führte zu einer signifikant stärkeren Inhibition der HPV im Vergleich mit nicht-hypoxieinduzierten Vasokonstriktionen.

4. Durch die Untersuchung des Effektes von Guanylatcyclaseund Phosphodiesterase-Inhibitoren, im Vergleich von intakter und blockierter endogener NO-Synthese, konnte gezeigt werden, dass nur die NO-stimulierte cGMP-Bildung einen spezifischen Einfluss auf die Regulation der HPV hat. Somit konnten andere Guanylatcyclase-Stimulatoren, wie $\mathrm{H}_{2} \mathrm{O}_{2}$, $\mathrm{CO}$ und ANP als Mediatoren des HPV-eigenen Mechanismus ausgeschlossen werden. Diese Daten widerlegen, zumindest für Kaninchenlungen, die von anderen Arbeitsgruppen postulierte Vorstellung, dass die HPV über eine tonische normoxische $\mathrm{H}_{2} \mathrm{O}_{2}$-vermittelte Guanylatcyclase-Stimulierung, die unter Hypoxie verringert wird, gesteuert wird. Aufgrund der Daten der vorliegenden Schrift konnte geschlossen werden, dass NO unter physiologischen Bedingungen einen spezifischen Effekt auf die Regulation der akuten HPV ausüben kann, dass das pulmonale NO-System aber sicherlich nicht das alleinige und wesentliche Regulationsprinzip der hypoxischen pulmonalen Vasokonstriktion darstellt. Diese Schlussfolgerung bezieht sich auf die Tatsache, dass auch eine vollständige Blockade der pulmonalen NO-Synthese nicht in der Lage war, den Hypoxieeffekt unter normoxischen Bedingungen zu imitieren, und dass die HPV auch unter diesen Bedingungen voll reaktionsfähig war.

Die Experimente zum Metabolismus reaktiver Sauerstoffspezies und potenzieller $\mathrm{O}_{2}$-Sensoren erlaubten, folgende neue Vorstellung von der Regulation der HPV zu entwickeln: $\mathrm{O}_{2}$-Sensor ist eine nicht-leukozytäre NADPH-Oxidase, die unter Hypoxie (möglicherweise über einen Proteinkinase C-abhängigen Mechanismus) vermehrt Superoxid bildet. Steigende Superoxidkonzentrationen lösen dann, nach einer Superoxiddismutase-abhängigen Konversion des $\mathrm{O}_{2}^{-} \mathrm{zu} \mathrm{H}_{2} \mathrm{O}_{2}$, über einen noch nicht identifizierten Mechanismus die Vasokonstriktion aus. Dieses Konzept stützt sich auf folgende Ergebnisse:

1. Mit den NADPH-Oxidase-Inhibitoren Diphenyleneiodonium und 4-(2-Aminoethyl)benzensulfonylfluorid gelang es, die HPV spezifisch (im Vergleich mit nicht-hypoxieinduzierten 
Vasokonstriktionen) zu inhibieren. Die Inhibitoren imitierten den Hypoxieeffekt jedoch nicht, da sie unter Normoxie keine entsprechende Vasokonstriktion auslösten.

2. Durch den Proteinkinase C (PKC)-Inhibitor Bisindolylmaleimid I konnte die HPV ebenfalls spezifisch inhibiert werden, ohne den Hypoxieeffekt zu imitieren.

3. Nitroblautetrazolium (NBT), eine Substanz, die Superoxid bindet und somit die $\mathrm{H}_{2} \mathrm{O}_{2}$-Bildung verhindert, hatte, ebenso wie der SOD-Inhibitor Triethylentetramin, der die Konversion von $\mathrm{O}_{2}^{-} \mathrm{zu} \mathrm{H}_{2} \mathrm{O}_{2}$ hemmt und dadurch wie NBT die $\mathrm{H}_{2} \mathrm{O}_{2}$-Konzentration verringern sollte, eine spezifische Inhibition der HPV zur Folge. Auch diese Substanzen konnten unter Normoxie keine HPV imitieren. Es muss jedoch einschränkend festgehalten werden, dass zahlreiche dieser Ansätze auch mit der Hypothese kompatibel sind, dass eine vermehrte SauerstoffradikalFreisetzung aus der mitochondrialen Atmungskette unter Hypoxie der entscheidende Sensormechanismus der HPV sein könnte.

In dem letzten Abschnitt der Arbeit wurden Untersuchungen an isolierten pulmonalarteriellen glatten Muskelzellen und pulmonalarteriellen Fibroblasten vorgenommen. Diese Daten deuten auf eine wichtige Rolle von pulmonalarteriellen Fibroblasten für den pathophysiologischen Proliferationsprozess von pulmonalarteriellen glatten Muskelzellen unter Hypoxie hin und lassen auf eine wichtige Bedeutung des hypoxieinduzierbaren Transkriptionsfaktors HIF für diesen Prozess schließen. Möglicherweise eröffnen sich hier Möglichkeiten mit modernen molekularbiologischen Techniken neue therapeutische Perspektiven zur Behandlung der hypoxieinduzierten pulmonalen Hypertonie zu entwickeln.

Zusammenfassend weisen die Daten der Arbeit darauf hin, dass die akute hypoxische Vasokonstriktion (ausgelöst durch eine Hypoxiedauer von Sekunden bis wenigen Minuten), die für die Ventilations-Perfusions-Anpassung in der Lunge verantwortlich ist, und die pulmonalvaskuläre Konstriktion unter protrahierter (mehrstündiger) alveolärer Hypoxie prinzipiell gleichen Regulationsmechanismen unterliegen. Möglicherweise werden in der protrahierten Phase der hypoxischen Vasokonstriktion bereits die pathogenetischen Vorgänge induziert, die dann zu dem Gefäßumbauprozess unter chronischer alveolärer Hypoxie führen. Für den Regulationsmechanismus der akuten und protrahierten hypoxischen Vasokonstriktion konnte ein neues Konzept entwickelt werden, das eine vermehrte Bildung reaktiver $\mathrm{O}_{2}$-Spezies unter Hypoxie in den Mittelpunkt stellt. Darüber hinaus weisen die Daten der Arbeit darauf hin, dass pulmonalarteriellen Fibroblasten und dem Transkriptionsfaktor HIF für die morphologische Fixierung eines hypoxieinduzierten pulmonalen Hypertonus eine wesentliche Bedeutung zukommen könnte. Weitergehende Untersuchungen müssen zeigen, ob sich das erarbeitete Konzept zu dem $\mathrm{O}_{2}$-Sensor und -Signaltransduktionsmechanismus der akuten und protrahierten HPV bestätigen lässt, und ob dieses Konzept auch Gültigkeit für die initialen Regulationsprozesse des Gefäßumbaus unter chronischer Hypoxie hat.

\section{Cheyne Stokes Atmung bei kardialer Kachexie und Herzinsuffizienz}

Die Deutsche Atemwegsliga hat das diesjährige Forschungsstipendium für Atemwegserkrankungen an Dr. Gerrit Hagenah von der Universitätsklinik Göttingen, Abteilung für Kardiologie und Pneumologie, vergeben. Der von GlaxoSmithKline gestiftete Preis in Höhe von 12 500,- $€(\mathbf{2 5} \mathbf{0 0 0},-\mathbf{D M})$ wurde im Rahmen des 43. Kongresses der Deutschen Gesellschaft für Pneumologie in Bochum verliehen. „Es ist sehr begrüßenswert, dass es Unternehmen gibt, die sich für die Forschung stark machen und diese großzügig unterstützen“, erklärte Prof. Dr. Heinrich Worth, Fürth, Vorsitzender der Deutschen Atemwegsliga

Dr. Gerrit Hagenah forscht seit 1997 in der pneumologischen Arbeitsgruppe von Prof. Dr. med. S. Andreas an der Universitätsklinik Göttingen zum Thema Cheyne-Stokes-Atmung (CSA) und Herzinsuffizienz. 1998 promovierte er mit seiner Arbeit „Über Herzrhythmusstörungen und nächtliche Sauerstofftherapie bei herzinsuffizienten Patienten mit CSA und über Schlafänderungen als Auslöser der Cheyne-Stokes-Atmung“. Die CSA wird grundsätzlich als prognostisch schlechtes Zeichen gewertet und tritt häufig nachts bei Patienten mit Herzinsuffizienz auf. Charakteristisch sind eine Abnahme der arteriellen Sauerstoffsättigung bei einer gleichzeitigen Dämpfung des Atemantriebs.

Patienten mit Herzinsuffizienz, die zugleich an einer kardialen Kachexie leiden, weisen eine deutlich schlechtere Prognose unabhängig von den kardialen Messparametern auf. Verschiedene Studien deuten darauf hin, dass eine Ursache dafür in einem gestörten Regelkreis der Atmung liegt. Ziel der jetzt geförderten Arbeit ist es, das Vorkommen der CSA bei herzinsuffizienten Patienten mit kardialer Kachexie zu ermitteln. Dabei sollen auch die Auswirkungen der CSA auf immunsystemische und neurohormonale Regulationsmechanismen mittels laborchemischer Messgrößen wie TNF- $\alpha$, Endothelin-1 und Interleukin- 6 erfasst werden. Davon abgeleitete neue Therapiemöglichkeiten könnten die körperliche Belastbarkeit und die Lebensqualität der betroffenen Patienten verbessern. 